\title{
Verdinglichung als Pathologie zweiter Ordnung
}

\author{
Von TITUS STAHL (Frankfurt/M.)
}

\begin{abstract}
I.
Der Begriff der „Verdinglichung“ ist kein Begriff, der aus unserer Alltagssprache stammt und dessen Bedeutung daher unmittelbar evident wäre. Er hat seinen Ursprung vielmehr in der Tradition kritischer Gesellschaftstheorie. Auf Grund des Pluralismus dieser Tradition lässt sich seine Bedeutung aber auch nicht durch einen Rückgriff auf eine von allen relevanten Theorien geteilte Definition klären. Deshalb muss vor der Diskussion der Vor- und Nachteile einzelner Verdinglichungstheorien die Frage gestellt werden, ob es überhaupt eine Kernbedeutung dieses Begriffs gibt, die den gemeinsamen Bezugspunkt aller Formen der Verdinglichungskritik darstellt.

Im weitesten Sinne soll „Verdinglichung“ zunächst heißen, dass etwas entweder zum Ding wird oder fälschlicherweise als Ding wahrgenommen oder behandelt wird, das ,eigentlich“ kein Ding ist. Wie sich schnell zeigt, verbergen sich jedoch in verschiedenen Anwendungsbereichen hinter dieser vagen Beschreibung höchst unterschiedliche Intuitionen. Es ist daher sinnvoll, einleitend vier Sphären zu unterscheiden, in denen dieser Begriff im Rahmen der Verdinglichungskritik Anwendung findet.

Die Unterscheidung zwischen Dingen und Nicht-Dingen kennt Verwendungsweisen, die primär auf einen normativen oder sogar moralischen Unterschied abzielen. ${ }^{1}$ Dazu gehört erstens die Unterscheidung zwischen Dingen und Personen oder Subjekten. Wie die berühmten Formulierungen von Kant nahe legen ${ }^{2}$, stellt eine bestimmte Behandlung von Personen einen moralischen Kategorienfehler dar, während die gleiche Behandlung im Fall von Dingen unproblematisch ist. $\mathrm{Zu}$ den „Dingen“ muss daher nach solch einer normativ motivierten Unterscheidung all jenes zählen, das instrumentalisiert, verkauft, benutzt und zerstört werden darf ${ }^{3}$, während es verdinglichend ist, Personen so zu behandeln. „Verdinglichung“ heißt in dieser Bedeutung folglich, Personen so zu behandeln, als hätten sie den moralischen Status bloßer Dinge.

Damit verwandt ist ein zweiter Anwendungsbereich des Verdinglichungsbegriffs. Wenn von der Verdinglichung der eigenen Person gesprochen wird, ist gemeint, dass Personen ihre eigenen Fähigkeiten, Emotionen oder Werthaltungen als unverrückbar vorgegeben oder als
\end{abstract}

1 Vgl. Honneth (2005), 16.

2 Kant (1977), 60.

3 Nussbaum (2002). 
instrumentalisierbar behandeln. Auch wenn es so scheint, als ob solche Verhaltensweisen nur einen weiteren Bereich der Verdinglichung von Personen darstellen, ist dieses Phänomen, das Lukács eindrucksvoll am Beispiel des Journalismus schildert ${ }^{4}$, weniger ein im engeren Sinne moralisches als vielmehr ein ethisches Problem ${ }^{5}$ : Der Schaden, der durch solche Haltungen verursacht wird, besteht nicht in einem Bruch moralischer Normen, sondern darin, dass wir eine schlechte, verkümmerte Haltung zu uns selbst einnehmen.

Mit diesen beiden Feldern hat sich der Anwendungsbereich des Verdinglichungsbegriffs jedoch keineswegs erschöpft: In der Tradition kritischer Gesellschaftstheorie geht es - wie in vielen Formen alläglicher Verdinglichungskritik - nicht primär um die fehlerhafte Behandlung anderer Personen oder der eigenen Persönlichkeit. Vielmehr steht hier ein anderer, dritter Bereich im Mittelpunkt: Wenn soziale oder geistige Prozesse und Beziehungen als Dinge - das heißt, als objektiv gegeben, als unveränderlich oder als menschenunabhängig - in einer sozialen oder historischen Praxis auftreten oder wahrgenommen werden, dann ist eine solche Praxis kritikwürdig.

In diesem Sinne betreibt Marx in dem Kapitel über den „Fetischcharakter der Ware“ im Kapital Verdinglichungskritik. Im Kapitalismus, so Marx, präsentieren sich gesellschaftliche Beziehungen in der Form von Eigenschaften der Waren, also von dinglichen Qualitäten. Dabei ist es

„nur das bestimmte gesellschaftliche Verhältnis der Menschen selbst, welches hier für sie die phantasmagorische Form eines Verhältnisses von Dingen annimmt" . ${ }^{6}$

Diese Kritik an der Verdinglichung von Prozessen oder Beziehungen ist nicht nur bei den gesellschaftstheoretischen Klassikern zu finden. Es stellt schon fast eine alltägliche Form politischer Aufklärung dar, wenn Kritikerinnen und Kritiker darauf hinweisen, dass bestimmte gesellschaftliche Prozesse nicht natürlich oder unvermeidbar sind. Im gleichen Sinne ist die Kritik daran zu verstehen, dass Entitäten, die nur durch Beziehungen und gesellschaftliches Handeln existieren (etwa Staat, Gesellschaft oder Volk), so repräsentiert oder praktisch behandelt werden, als handle es sich um menschenunabhängige Gegenstände oder Akteure.

Sein viertes Anwendungsgebiet findet der Verdinglichungsbegriff überraschenderweise bei den Dingen selbst: Nicht erst Lukács versteht den Prozess der Verdinglichung als eine Form der Veränderung unserer Erfahrung gewöhnlicher Objekte. ${ }^{7}$ Es gehört vielmehr zu den kulturkritischen Gemeinplätzen der Moderne, dass ein Prozess der Verdinglichung beklagt wird, der die Entqualifizierung von Erfahrungen, die Abwesenheit eines übergreifenden Zusammenhangs sinnvoller Beziehungen zwischen natürlichen Gegenständen und Prozes-

4 „Der spezialistische, Virtuose`, der Verkäufer seiner objektivierten und versachlichten geistigen Fähigkeiten, [...] gerät auch in eine kontemplative Attitüde zu dem Funktionieren seiner eigenen, objektivierten und versachlichten Fähigkeiten. Am groteskesten zeigt sich diese Struktur im Journalismus, wo gerade die Subjektivität selbst, das Wissen, das Temperament, die Ausdrucksfähigkeit zu einem [...] eigengesetzlich in Gang gebrachten Mechanismus wird.“ (Lukács 1968, 275)

5 Wie die Debatte um Leihmutterschaft (vgl. Anderson 1990) zeigt, ist die Abgrenzung zwischen moralischen und ethischen Fragestellungen auch hinsichtlich der Verdinglichung anderer Personen nicht eindeutig.

6 Marx (1962), 86.

7 „Diese rationelle Objektivierung verdeckt vor allem - den qualitativen und materiellen - Dingcharakter aller Dinge. Indem die Gebrauchswerte ausnahmslos als Waren erscheinen, erhalten sie eine neue Objektivität, eine neue Dinghaftigkeit, [...] in der ihre ursprüngliche, eigentliche Dinghaftigkeit vernichtet wird, verschwindet.“(Lukács 1968, 267; Fehler im Original) 
sen sowie die Dominanz einer quantifizierenden und formalen Perspektive auf Erfahrungsgegenstände zur Folge hat. ${ }^{8}$ Diese Aspekte betreffen die Form, in der Dinge erfahren werden, auftreten oder existieren. Wenn wir auch diese Phänomene als „Verdinglichung“ beschreiben wollen, muss damit in diesem Fall etwas anderes gemeint sein als nur eine Fehlwahrnehmung von Akteuren oder Prozessen als unbelebte Objekte.

Wie dieser Durchgang durch einige Anwendungsgebiete des Begriffs der Verdinglichung zeigt, handelt es sich um eine äußerst heterogene Zusammenstellung von Phänomenen, von Standards der Kritik und vermutlich auch von ontologischen und methodologischen Hintergrundannahmen. ${ }^{9}$ Dass sich der Begriff der „Verdinglichung“ dazu eignet, einen Bereich von zusammenhängenden pathologischen Erscheinungen nach klaren Kriterien abzugrenzen, ist daher keineswegs offensichtlich, sondern erfordert eine Begründung.

Es stellt sich insbesondere die Frage, welche Normen die Verdinglichungskritik verwendet. Die Verdinglichung sozialer Verhältnisse, die Illusion ihrer Unveränderbarkeit und Naturgegebenheit kann als falsche Überzeugung oder kognitive Haltung, also als Form eines Irrtums analysiert werden. Dies legt nahe, Verdinglichungskritik als eine Form epistemischer Kritik zu verstehen. ${ }^{10}$ Bei Marx ${ }^{11}$ und Lukács ${ }^{12}$ hingegen scheinen falsche Überzeugungen eine Folge gesellschaftlicher Verdinglichungsprozesse zu sein, die keinen primär epistemischen Charakter haben. Ihre Verdinglichungskritik kann sich daher nicht auf die Maßstäbe der Wahrheit oder Rechtfertigung von Überzeugungen beziehen, sondern muss andere, praktische Normen zur Grundlage haben.

Auch die Verdinglichung anderer Personen und des eigenen Selbst sind kaum in das Raster einer epistemischen Kritik integrierbar, denn es handelt sich bei diesen Phänomenen offensichtlich nicht um das Produkt falscher Überzeugungen im engeren Sinn. Eine Verdinglichungstheorie, die es uns erlaubt, alle vier genannten Anwendungsbereiche des Begriffs zu verstehen und die klassischen Ansätze zu rekonstruieren, muss Verdinglichung daher zumindest in manchen Fällen als eine Verletzung nicht-epistemischer, praktischer Normen verstehen.

Dabei handelt es sich, wie schon in der vorausgegangenen Diskussion deutlich wurde, nicht primär um moralische Normen. Da alle Formen der Verdinglichung das Phänomen involvieren, dass Personen bestimmte pathologische Formen des Umgangs mit anderen, sich selbst und der objektiven und sozialen Realität entwickeln, müssen die in Frage kommenden Normen vielmehr Normen sein, die die „richtigen“ Formen solcher Praktiken von pathologischen Verzerrungen zu unterscheiden erlauben.

Spätestens bei der Frage, was dies heißen kann, scheiden sich die Geister. Deshalb soll im Folgenden zunächst am Beispiel von Lukács' Verdinglichungstheorie verdeutlicht werden, dass er einen solchen Begriff richtiger Praxis in den Mittelpunkt stellt (II). Es wird sich dabei zeigen, dass seine Konzeption des Zusammenhangs zwischen Praxis und Verdinglichung heute nicht mehr überzeugen kann. Daher werde ich im dritten Teil des Aufsatzes eine alternative Theorie sozialer Praktiken skizzieren, die Verdinglichungsprozesse als Pathologien zweiter Ordnung, als Pathologien der Reflexivität und der praktischen Zugänglichkeit sozialer Prak-

\footnotetext{
8 Vgl. beispielsweise die Darstellung dieser Entwicklung in: Taylor (1983), Kap. 1.

9 Vgl. Jaeggi (2003).

10 So beschreiben etwa Peter L. Berger und Thomas Luckmann Verdinglichung als eine „Modalität des Bewusstseins" (Berger u. Luckmann 1977, 96); vgl. auch Berger u. Pullberg (1962), dazu kritisch Pitkin (1987).

11 Vgl. etwa die Luft-Analogie in: Marx (1962), 88.

12 Lukács (1968), 261.
} 
tiken, rekonstruiert (III). Abschließend soll untersucht werden, welche normativen Ressourcen ein solches Modell einer kritischen Theorie an die Hand gibt (IV).

\section{II.}

1. Es kann als die bleibende Leistung von Lukács' Aufsatz Die Verdinglichung und das Bewusstsein des Proletariats gelten, dass er systematisch den Zusammenhang der unterschiedlichen Formen von Verdinglichungserfahrungen zu begründen versucht.

Lukács geht zwar von der Marxschen Theorie vom Fetischcharakter der Warenform aus. Auf der Grundlage sowohl der Weberschen Rationalisierungsthese als auch Simmels Theorie der Moderne erweitert er sie aber schnell von einer im engeren Sinne ökonomie- und ideologiekritischen Theorie zur einer umfassenden Theorie sozialer und kultureller Verdinglichung.

Die zunehmende Einbindung aller sozialen Prozesse in den kapitalistischen Markt, die dadurch erfolgende Rationalisierung aller Tätigkeiten und die wachsende Warenförmigkeit sozialer Beziehungen führt nach Lukács in allen gesellschaftlichen Bereichen zur Dominanz dessen, was er eine verdinglichte „Gegenständlichkeitsform“ nennt. ${ }^{13}$ Diese Entwicklung ist dadurch gekennzeichnet, dass die organische Einheit sowohl von Objekten als auch Subjekten zerstört wird ${ }^{14}$, dass die innere und äußere Welt eine unzugängliche Objektivität erhält und zu einem scheinbar durch eherne Gesetze bestimmten, unveränderlichen Mechanismus wird. Auf der Seite der ihr unterworfenen Menschen produziert diese Entwicklung die Haltung der „Kontemplation“, der kalkulierenden Anpassung an ein System, dem sie als einflusslose Zuschauer machtlos unterworfen sind. ${ }^{15}$ Diese Haltung dehnt sich im Zuge der gesellschaftlichen Rationalisierung des inneren und äußeren Lebens auf alle Bereiche der Person, der Natur und der Gesellschaft aus. Im Verlauf dieses Prozesses tritt eine Vielzahl objektiver und subjektiver Verdinglichungsphänomene auf, die aber alle als Aspekte dieser grundlegenden Pathologie analysierbar sind.

2. Durch die Entwicklung einer umfassenden Verdinglichungstheorie kann Lukács vier Leistungen erbringen: Er kann erstens scheinbar disparate Formen kultureller und sozialer Pathologien als Aspekte eines einheitlich theoretisch erfassbaren, umfassenden Phänomenkomplexes analysieren. Er kann zweitens diese Pathologien kausal auf eine Eigenschaft kapitalistischer Wirtschaftsordnungen zurückführen. Er kann damit drittens eine Erklärung für das zunächst verblüffende Phänomen vorlegen, dass Verdinglichungserfahrungen eine Besonderheit moderner Gesellschaften sind, obwohl sich diese Gesellschaften durch ein gesteigertes Potenzial der Reflexion auf ihre eigene Konstitution auszeichnen. Dadurch kann er eine nostalgische Sichtweise auf eine scheinbar unverdinglichte Vergangenheit vermeiden, die unplausibel ist, wenn erklärt werden soll, wieso gerade die Moderne in besonderem Maße von ideologischen Kategorienfehlern geprägt sein soll. ${ }^{16}$ Lukács lässt schließlich viertens die Verdinglichungstheorie reflexiv werden, da er die Verdinglichung nicht nur als Quelle sozialer

13 Ebd., 258; zu Weber und Simmel vgl. Dannemann (1987), Kap. III u. IV.

14 Lukács (1968), 262 ff.

15 Ebd., 264.

16 Auch wenn die Kausalthese nicht überzeugt (Honneth 2005, 94 ff.), versucht Lukács zumindest, die Leistung zu erbringen, die Habermas Marx abverlangt: „die Zerstörung traditionaler Lebensformen von der Verdinglichung posttraditionaler Lebensweisen“ zu unterscheiden (vgl. Habermas 1995, Bd. 2, 501). 
Pathologien begreift. Auch die Unfähigkeit der traditionellen Philosophie und Wissenschaft, diese Pathologien gedanklich zu durchdringen, wird als Ausdruck einer verdinglichten Haltung zur Welt erklärt. ${ }^{17}$

Diese Leistungen erklären, wie der Verdinglichungsbegriff zum „Paradigmakern“ kritischer Theorien werden konnte. ${ }^{18}$ Die gesamte Tradition der kritischen Theorie wird durch die Idee geprägt, dass die Form, die gesellschaftliche Beziehungen im Warentausch annehmen, durch den Rationalisierungsdruck kapitalistischer Prozesse in ihrer quantifizierenden, entfremdenden und letztlich herrschaftsförmigen Natur alle Bereiche des gesellschaftlichen, kulturellen und psychischen Lebens kolonisiert und schließlich die Rationalität der Subjekte zum Instrument ihrer eigenen Beherrschung macht.

3. Dass die Verdinglichungskritik keine epistemische Kritik, sondern eine Kritik an dem Fehlschlagen einer bestimmten gesellschaftlichen Praxis ist, der als Gegenbild die Vorstellung einer besseren, gelingenden Praxis gegenübersteht ${ }^{19}$, kann an drei argumentativen Figuren des Verdinglichungsaufsatzes nachgewiesen werden: Wenn Lukács die subjektive Seite der Verdinglichung als „kontemplative Haltung“ beschreibt, bezieht er sich dabei erstens auf eine Einstellung, die eine gelingende Art der praktischen Bezugnahme verhindert. ${ }^{20}$ Zweitens betont er, dass die Lösung der Probleme der klassischen Philosophie, insbesondere des Irrationalitätsproblems, in der Praxis zu suchen sei. ${ }^{21}$ Drittens kann nach seiner Darstellung die Herrschaft der Verdinglichung nur überwunden werden, wenn sich eine neue Form der Praxis entwickelt $^{22}$, die in der Lage ist, die Gegenständlichkeitsformen zu verändern. ${ }^{23}$

Es muss Lukács also bei der Kritik der Verdinglichung primär um die Kritik einer Form von Praxis gehen: Verdinglichte Praxisformen produzieren ein Bewusstsein, das Subjekten keinen - oder nur einen verzerrten Zugang - zu ihrer Tätigkeit und zu ihrem praktischen Verhältnis zur Welt erlaubt, und das daher zu einer „,kontemplativen“ Haltung führt.

4. Lukács kann aber nur deshalb eine so umfassende Verdinglichungstheorie entwickeln, weil er sie vor dem Hintergrund einer ebenso umfassenden Theorie menschlicher Praxis entwirft. Diese Praxis stellt er sich nach dem Vorbild der Entäußerung von Subjekten vor, das er aus dem deutschen Idealismus gewinnt. ${ }^{24}$ Die moderne Rationalisierung und die Verdinglichung stellen ein Scheitern einer solchen Praxis dar, weil sie dazu führen, dass die Akteure dieser Praxis ein pathologisches Verhältnis zur Realität entwickeln, das ihnen nicht erlaubt, sich darüber bewusst zu werden, dass ihr eigenes Handeln diese Realität konstituiert. ${ }^{25}$ Erst wenn das Proletariat, als derjenige Teil der Gesellschaft, der einerseits von jeder Illusion der Selbsttätigkeit abgeschnitten und bloßes Objekt der gesellschaftlichen Prozesse, andererseits aber faktisch das

17 Lukács (1968), 187 ff.

18 Brunkhorst (1983).

19 So auch Honneth (2005), 25.

20 Lukács (1968), $304 \mathrm{f}$.

21 So schreibt er zu Kant: „Daß Kant damit - freilich in einer negativen, entstellenden Weise [...]hier gerade die Struktur der wahren Praxis als der Überwindung der Antinomien des Seinsbegriffs beschreibt, ist sowohl ihm selbst wie den Kritikern [...] entgangen." (Ebd., 305)

22 „Ihre Aufhebung [...] muß sich zu ihrer praktischen Aufhebung als Lebensformen der Gesellschaft erheben." (Ebd., 362, vgl. auch 384)

23 Ebd., $384 \mathrm{f}$.

24 Arato u. Breines (1979), 130.

25 Zur Kritik daran vgl. Habermas (1995), Bd. 1, 474 ff. 
Subjekt aller gesellschaftlichen Produktion und Konstitution ist, sich seiner kollektiven Subjektrolle bewusst wird und diese praktisch realisiert, und wenn es ein Verhältnis zur „Totalität“ der Gesellschaft gewinnt ${ }^{26}$, wird die verdinglichte Gegenständlichkeitsform überwunden.

Hinter diesem Begriff von Praxis steht die Vorstellung einer - wenn auch nicht abschließbar gedachten ${ }^{27}$ - Entwicklung eines Kollektivsubjekts, das in einem historischen Prozess die Gegenständlichkeitsformen durch seinen Bezug auf die Totalität von der Verdinglichung befreit und damit zugleich auch die Probleme traditioneller philosophischer Theorien löst. Diese Vorstellung ist aber nicht nur eine radikale Übersteigerung eines hegelianischen Geschichtsbildes, sondern treibt auch ein an Fichte geschultes Bild von Subjektivität und Objektivität auf die Spitze, das im Lichte heutiger philosophischer Einsichten nicht mehr akzeptabel ist. ${ }^{28}$

Aber auch abgesehen von seinen theoretischen Voraussetzungen ist dieses Modell für die Gesellschaftskritik unproduktiv. Seine geschichtsphilosophische Perspektive bietet keinen Ansatz für konkrete Verdinglichungskritik an spezifischen sozialen Praktiken. Auch auf die Frage, wie eine Grenze zwischen der praktisch notwendigen alltäglichen Distanzierung von den Interaktionspartnern und Objekten des eigenen Handelns und tatsächlich pathologischen Verdinglichungsphänomenen gezogen werden könnte, hat dieses Modell keine Antwort zu bieten.

5. Aus diesen Einwänden ergibt sich das Dilemma, das die folgenden Überlegungen beschäftigen soll: Wenn die Verdinglichungstheorie nicht ein Oberbegriff für unverbundene Überlegungen zur angewandten Ethik, sondern Teil einer gesellschaftskritischen Theorie sein soll, kann sie dies nicht auf der Grundlage des Lukácsschen Praxiskonzepts sein. Der Wegfall dieser Basis scheint jedoch zwangsläufig dazu zu führen, dass die Rechtfertigung für die umfassende Anwendung eines einheitlichen Verdinglichungsbegriffs schwindet.

Eine Rekonstruktion der Verdinglichungstheorie müsste daher versuchen, eine Konzeption sozialer Praxis zu entwickeln, die die bereits genannten Leistungen der Lukácsschen Theorie erbringen kann. Insbesondere müsste sie es erlauben, den systematischen Zusammenhang zwischen der Verdinglichung von sozialen Beziehungen und Prozessen, der Verdinglichung von anderen Personen, der Verdinglichung der eigenen Person und der Verdinglichung der Dinge plausibel zu machen. Darüber hinaus müsste sie eine überzeugende Antwort auf die Frage anbieten, wieso gerade die Struktur moderner Gesellschaften und moderner Subjektivitätsformen in so hohem Maße mit Verdinglichungsphänomenen einhergeht.

6. Die bekanntesten Reaktualisierungsversuche der Verdinglichungstheorie - Jürgen Habermas' Theorie des kommunikativen Handelns ${ }^{29}$ und Axel Honneths Tanner Lectures ${ }^{30}$ - versuchen in genau diesem Sinne, die geschichtsphilosophisch aufgeladene und normativ unklare Praxiskonzeption von Lukács durch andere Modelle sozialer Praxis zu ersetzen und den Verdinglichungsbegriff auf ihrer Grundlage zu rekonstruieren. Während bei Habermas das kommunikative Handeln den Hintergrund bildet, vor dem die Kolonisierung der Lebenswelt durch strategische Einstellungen als Pathologie kritisiert werden kann, ist es bei Honneth die

26 Vgl. seine Bemerkungen zum Individuum in: Lukács (1968), 380; zur Totalität vgl. ebd., 384; vgl. auch Jay (1984), $108 \mathrm{f}$.

27 Lukács (1968), 358, 384, 394.

28 Honneth (2005), 16.

29 Habermas (1995), zur Kritik an Lukács vgl. Bd. 1, 474 ff., zum Rekonstruktionsvorschlag vor allem Bd. 2, 278, zum Zusammenhang mit Verdinglichung 476.

30 Erschienen als Honneth (2005). 
Haltung basaler Anerkennung in unseren intersubjektiven Beziehungen, deren Verdrängung oder Verzerrung zu Verdinglichungsphänomenen führt.

Von beiden Autoren wird aber nicht nur Lukács' starker Praxisbegriff aufgegeben, sondern auch der Anwendungsbereich der Verdinglichungskritik beschränkt ${ }^{31}$ : Bei Habermas müssen notwendigerweise Praktiken, die für Lukács zentral sind - etwa die materielle Reproduktion und der Staat -, aus dem Bereich der verdinglichungstheoretisch kritisierbaren Phänomene herausfallen, soweit Habermas sie als legitime Sphären systemischer Handlungskoordination versteht. Auch bei Honneth wird unklar, ob seine Theorie der „Anerkennungsvergessenheit“ zur Kritik der Verdinglichung von Objekten und der sozialen Realität als Ganzer dienen $\mathrm{kann}^{32}$, weil er seinen Praxisbegriff relativ eng auf intersubjektive Beziehungen zuschneidet.

\section{III.}

1. Die Reaktualisierungsversuche zeigen, dass die Antwort auf die Frage, ob es möglich ist, eine Theorie der Verdinglichung zu entwickeln, die Lukács' umfassenden Anspruch aufrechterhält, entscheidend davon abhängt, welcher Begriff der Praxis vorausgesetzt wird. Daher soll im Folgenden versucht werden, einen alternativen Praxisbegriff zu skizzieren, der in der Lage ist, einen umfassenden Verdinglichungsbegriff zu tragen, ohne die unplausiblen Konsequenzen des Lukácsschen Modells mit sich zu bringen.

Was kann es aber heißen, so umfassend von sozialen Praktiken zu sprechen, dass alle relevanten Aspekte von Verdinglichungserfahrungen damit erfasst werden können?

Es lohnt sich an diesem Punkt, die Debatte zur Kenntnis zu nehmen, die sich in der Nachfolge Wittgensteins in der Philosophie und Soziologie entwickelt hat. ${ }^{33}$ In dieser Theorietradition wurde nämlich versucht, einen Begriff sozialer Praxis zu entwickeln, der nicht auf Phänomene der Kommunikation oder der Moral beschränkt ist: Soziale Praktiken sind - so könnte man, freilich verkürzt, diesen Vorschlag beschreiben - systematische Handlungszusammenhänge, die durch sozial etablierte, normative Standards reguliert werden. Die dabei wirksamen expliziten Regeln und impliziten Verhaltensorientierungen beruhen auf der Seite der partizipierenden Subjekte auf dispositional eingespielten Fähigkeiten zum situationsgerechten Handeln. Die Struktur einer Praxis wird jedoch nicht durch solche Dispositionen erschöpft, sondern auf der Seite der sozialen Interaktion durch intersubjektive Prozesse ergänzt, durch die Normen ausgehandelt und Differenzen in der Anwendung von Regeln in die Praxis reintegriert werden. ${ }^{34}$

In solchen Praktiken bildet die Gesamtheit der für die Praxis relevanten Objekte, Handlungen und Personen den Kontext, in dem eine bestimmte Reaktion oder Handlung eines Subjekts intersubjektiv als angemessen oder unangemessen gelten kann. Je nachdem, welche Normen in einer Praxis maßgeblich sind, werden daher andere Eigenschaften von Gegenständen oder Handlungen normativ relevant. Das heißt, dass die Zuschreibung von Eigenschaften an Handlungen, Personen und Objekte immer in einem Zusammenhang mit normativ auf-

31 Für eine ähnliche Kritik vgl. Chari (2010).

32 Vgl. Honneth (2005), 78.

33 Zu dieser Verwendungsweise des Begriffs Praxis vgl. Schatzki (1996), Schatzki u. a. (2001), Reckwitz (2003).

34 Das hier gemeinte Praxiskonzept trifft sich dabei eng mit dem Vorschlag von Bourdieu (1976); für eine an Wittgenstein orientierte Perspektive vgl. Bloor (1997). 
geladenen Unterscheidungen steht, die ihren Sinn aus der Funktion beziehen, die sie in der Regulation der Praxis spielen.

Wir können beispielsweise die Bedeutung von Eigenschaften wissenschaftlicher Veröffentlichungen, von Kunstwerken oder handwerklichen Produkten nur dann voll begreifen, wenn wir verstehen, dass die Zuschreibung dieser Eigenschaften bestimmte Handlungen normativ angemessen oder unangemessen macht und bestimmte Bewertungen anderer, vorangegangener Handlungen legitimiert. Die Zuschreibung solcher Eigenschaften ist daher nur dann verständlich, wenn wir auch die normativen Unterscheidungen nachvollziehen können, die die Praxis als Ganze strukturieren ${ }^{35}$ : In diesem Sinne gehört etwa die Unterscheidung zwischen Feigheit, Mut und Übermut zu einer Praxis militärischer Bewährung, die Unterscheidung zwischen Glaube und Aberglaube zu einer Praxis der Religion und die Unterscheidung zwischen handwerklichem Können und bloßem Ausführen zu einer Praxis der Produktion.

Um Missverständnisse zu vermeiden, muss betont werden, dass solche primär praktischen Unterscheidungen keineswegs immer diskursiven Ausdruck finden müssen. In fast allen komplexen Praktiken finden sie de facto jedoch in einem Vokabular Ausdruck, das wir mit Charles Taylor ein Vokabular kontrastiver Unterscheidungen nennen können. ${ }^{36}$

2. Zumindest ein Teil der Bedeutung der in einer Praxis relevanten Eigenschaften von Gegenständen (aber auch Handlungen, Personen und so weiter) wird also durch diese Praxis selbst festgelegt. Ohne diesen Ausdruck schon analysiert zu haben, könnte daher vermutet werden, dass die Form der so konstituierten Gegenstände, also die Weise, in der sie in ihrer jeweils relevanten Bedeutung konstituiert werden, in engem Zusammenhang zu der Form der entsprechenden Praxis steht. Wenn dies plausibel gemacht werden kann, könnte - wiederum, ohne diese Begriffe bereits erklärt zu haben - versucht werden, die Rede von der pathologischen Gegenständlichkeitsform der Verdinglichung in ein Vokabular pathologischer Praxisformen zu übersetzen.

Vor diesem Hintergrund stellt sich die Frage neu, in welchem Sinne wir Verdinglichung als eine Pathologie sozialer Praktiken verstehen können. Klarerweise kann es sich bei einer solchen Pathologie nicht um einen kontingenten Fehler der einen oder anderen Praxis handeln, sondern es muss ein strukturelles Problem angenommen werden, das soziale Praktiken (oder zumindest eine Klasse sozialer Praktiken) als solche betreffen kann. Nur unter dieser Annahme kann die Lukácssche These verständlich gemacht werden, dass es sich bei der Verdinglichung im Tausch, der Bürokratie und der Produktion um das gleiche Phänomen handelt, und dass Verdinglichungsformen in einer Sphäre Verdinglichung in anderen Sphären systematisch begünstigen können.

Es muss sich bei der Verdinglichung also um allgemeinere Strukturen sozialer Praktiken handeln, die dazu führen, dass die Mitglieder diese Praktiken und die durch sie konstituierten Beziehungen und Eigenschaften ,verdinglichen“, dass sie gezwungen sind, ihnen gegenüber kontemplative, machtlose, rein strategische oder objektivierende Haltungen einzunehmen.

Die zentrale These, die ich im Weiteren begründen möchte, besteht in der Behauptung, dass die „Form“ einer sozialen Praxis - und damit auch die Verdinglichung sozialer Praktiken - durch ihre Normen zweiter Ordnung konstituiert wird. Darunter verstehe ich diejenigen Normen, die festlegen, wie sich Personen in einer sozialen Praxis auf die Normen dieser Praxis praktisch beziehen können.

35 Vgl. Taylor (1975).

36 Vgl. ders. (1992). 
Dies ergibt sich aus folgender Überlegung: In sozialen Praktiken unterliegen im Normalfall nicht nur Handlungen erster Ordnung Normen - also etwa in der Praxis des Handwerks die physischen Handlungen produktiver Arbeit, in der Praxis des Militärs das kriegerische Handeln und so weiter. In jeder komplexen Praxis kann auch immer die Praxis selbst im Rahmen der Praxis zum Gegenstand eines Handelns zweiter Ordnung werden. Damit ist Folgendes gemeint: Handwerkliche Praktiken enthalten immer auch Normen, die die Handlungen regulieren, mit denen die Normen des Handwerks weiterentwickelt, kontrolliert, weitergegeben oder thematisiert werden, militärische Praktiken beinhalten in vielen Fällen Normen, die eine besondere Behandlung der militärischen Normen vorschreiben, und künstlerische Praktiken sind in der Moderne offensichtlich immer auch praktisch auf ihre eigenen Normen bezogen. Diese Handlungen, also die Veränderung, Weitergabe, Thematisierung, Konservierung oder Negierung von Normen, sind Handlungen, die sich auf Normen beziehen. Im Normalfall sind nicht alle solche Handlungen im Kontext einer Praxis legitim. Dies zeigt, dass sie von weiteren Normen reguliert werden - von Normen zweiter Ordnung.

Man könnte nun sagen, dass eine Praxis, sobald sie solche Normen zweiter Ordnung besitzt, eine bestimmte Form annimmt: Je nachdem, welche Normen zweiter Ordnung etwa die Praxis der gekonnten Herstellung eines Musikinstruments besitzt, kann sie die Form rationell-technischer Produktion, traditionsgebundenen Zunftwesens oder künstlerischer Virtuosität annehmen. Jede dieser Formen ist durch eine jeweils eigene Weise gekennzeichnet, in der die Mitglieder der Praxis auf ihre Normen handelnd Bezug nehmen können.

Wenn wir die „Form“ einer sozialen Praxis also mit der Gesamtheit ihrer Normen zweiter Ordnung identifizieren, ist der Schritt nicht mehr weit zu dem bereits geäußerten Verdacht, dass es sich bei den Veränderungen der Formen, die Lukács diagnostiziert, um Veränderungen auf der Ebene der Normen zweiter Ordnung handeln muss.

3. Bei der Verdinglichung handelt es sich um das Scheitern einer bestimmten Aneignung einer Praxis durch ihre Teilnehmer. Sofern dieses Scheitern eine systematische Ursache in der Form der Praxis haben soll, muss es durch die interne Struktur dieser Praxis, also gerade durch die Normen zweiter Ordnung, verursacht werden, die festlegen, wie sich die Mitglieder der Praxis auf ihre Normen beziehen können.

Eine verdinglichte Praxis muss also so verfasst sein, dass zwei Kriterien erfüllt sind: Erstens muss eine bestimmte Form der Aneignung oder Behandlung der Normen der Praxis eine „Gelingensbedingung“ dieser Praxis darstellen. ${ }^{37}$ Zweitens muss aber gerade die reflexive Struktur der Praxis, wie sie durch ihre Normen zweiter Ordnung gegeben ist, eine solche Aneignung verhindern. Das Scheitern eines erfolgreichen Bezugs auf die Praxis, das in dieser Situation notwendigerweise eintreten muss, führt dazu, dass die Mitglieder dieser Praxis sich nicht mehr erfolgreich die Bedeutung derjenigen Eigenschaften von Objekten, Handlungen und Personen in ihrem Bezug auf die Praxis vergegenwärtigen können, die ihren Sinn erst im Rahmen dieser Praxis erhalten.

Um diesen Vorschlag weiter zu konkretisieren: Eine komplexe soziale Praxis, die in diesem Sinne nicht nur reflexiv ist, sondern deren Regeln von ihren Mitgliedern auch Reflexivität einfordern, ist immer dann defizitär, wenn ihre Normen zweiter Ordnung ein Verhalten erzwingen, das systematisch den Versuch untergräbt, die praxiskonstitutiven Normen als praxiskonstituierte Normen zu behandeln.

37 Damit ist gemeint, dass die Normen der Praxis eine Orientierung zur Teilnahmebedingung machen, die auf eine solche Aneignung abzielt; vgl. die parallel laufende Idee von Timo Jütten in dieser Ausgabe der Deutschen Zeitschrift für Philosophie. 
Als ein Beispiel für diese Struktur kann die Praxis des Markttauschs gelten, die einerseits vorgibt, eine Sphäre individueller Autonomie zu verkörpern, die aber andererseits - nicht nur nach der Vorstellung von Lukács - eine praktische (das heißt unter anderem auch deliberative) Bezugnahme auf die Normen der Marktorganisation nicht als Handlung vorsieht. Die Struktur kapitalistischer Märkte, so eine verbreitete Kritik, verhindert nicht nur Reflexivität in der Praxis des Markttauschs selbst, sondern nötigt seine Teilnehmerinnen und Teilnehmer sogar, auch Versuche, in benachbarten Praktiken die soziale Konstitution seiner Normen praktisch zu realisieren, als Marktverzerrung zu behandeln. ${ }^{38}$ Genauso können wir etwa an Bildungspraktiken denken, die objektivierte Vorstellungen von „Begabung“ und „Leistung“" so festschreiben, dass eine Orientierung an der angeblichen Objektivität dieser Kriterien unabdingbar für die Partizipation an der Praxis ist. Auf Grund der dadurch etablierten Form der Praxis muss ein auf die Veränderung dieser Kriterien gerichtetes Handeln als regelwidrig oder gar unsinnig auftreten. ${ }^{39}$

Diese Beispiele verdeutlichen, inwiefern eine solche Praxis fehlerhaft ist: Sie enthält einen Widerspruch zwischen einem Anspruch auf Reflexivität und Normen, die seine Erfüllung verhindern. Eine solche Praxis wird für ihre Mitglieder „dinghaft“, weil ihre Normen zweiter Ordnung es unmöglich machen, die in dieser Praxis durch soziale Regeln konstituierten Eigenschaften von Objekten, Handlungen und Personen anders zu behandeln als bloß natürliche Eigenschaften.

Wenn diese Eigenschaften - beispielsweise in einer militärischen Praxis der Mut einer Handlung, in einer Erziehungspraxis die „Begabung“ einer Person oder in einer künstlerischen Praxis die Originalität eines Werks - auf Unterscheidungen beruhen, die sich praktisch verselbstständigen, dann lassen sie sich nicht mehr als Teil der Praxis behandeln und hindern die Subjekte daran, sich in angemessener Weise auf die Praxis reflexiv zu beziehen.

Dabei handelt es sich nicht primär um das Ergebnis eines Irrtums. Wenn die Normen einer Praxis einen solchen Widerspruch systematisch generieren, dann nicht deshalb, weil Personen die Eigenschaften von Objekten als dinglich „missverstehen“. Die Eigenschaften werden vielmehr für sie objektiv zu „dinglichen“ Eigenschaften im Lukácsschen Sinne, weil ihre Praktiken ihnen de facto keine Möglichkeit bieten, die Normen, die diese Eigenschaften in ihrer Bedeutung bestimmen, legitimerweise als Teil der Praxis und damit als Teil des eigenen Handelns zu behandeln. Wenn den Subjekten aber die Produkte ihrer eigenen Tätigkeit in dieser Weise objektiviert und unzugänglich gegenübertreten, dann erklärt dies Erfahrungen der Zerrissenheit, der Machtlosigkeit und der Unverbundenheit.

4. Wenn die Verdinglichungskritik so bestimmt wird, lässt sie sich in ein Analyseraster einordnen, das von Christopher Zurn und Robin Celikates vorgeschlagen wurde ${ }^{40}$ : Zurn und Celikates unterscheiden bei den Pathologien, die kritische Theorien diagnostizieren, zwischen Pathologien erster und zweiter Ordnung. Pathologien erster Ordnung sind konkrete Fehler oder Verletzungen von Normen, also etwa Fälle von falschen Überzeugungen, Ungerechtigkeiten oder Ähnlichem. Pathologien zweiter Ordnung hingegen sind diejenigen Pathologien,

38 Dies scheint mir etwa die Intuition zu sein, die hinter der Unterscheidung zwischen,,sozial eingebetteten" und selbstregulierenden Märkten bei Polanyi (1978) steht.

39 Ohne zu der damit verbundenen sachlichen Frage Stellung nehmen zu wollen, ist es in diesem Zusammenhang von Interesse, dass Gould (1994) die wissenschaftliche Entwicklung des IQ explizit als „reification“ bezeichnet (ebd., 295).

40 Zurn interpretiert den reflexiven Zugriff auf Praktiken jedoch weitgehend epistemisch (vgl. Zurn 2006); auch Celikates nimmt als Ursachen der Unzugänglichkeit von Praktiken Reflexivitätsdefizite im engeren Sinn des Wortes an (Celikates 2009). 
bei denen nicht oder nicht nur eine Norm verletzt wird, sondern bei denen die reflexiven Fähigkeiten von Subjekten untergraben werden, die es ihnen erlauben, zwischen Verletzung und Erfüllung von Normen zu unterscheiden, sich an Normen zu orientieren und die soziale Realität an diesen Normen zu messen.

Verdinglichungsphänomene zählen nach dieser Einteilung klarerweise zu den Pathologien zweiter Ordnung. Wenn Beziehungen, die ihren Sinn nur innerhalb einer Praxis haben, so auftreten, als seien sie von Natur aus gegeben, oder wenn die Teilnahme an einer Praxis nicht mehr als sozial reguliert, sondern als objektiv erzwungen wird, dann verhindert die „kontemplative“ Haltung, die sie zu diesen Beziehungen einnehmen müssen, eine reflexive Vergewisserung ihrer sozialen Veränderbarkeit und untergräbt damit die Autonomie der Subjekte.

Im Moment muss allerdings noch offen bleiben, ob der dadurch frustrierte Anspruch auf Reflexivität ein kontingentes Merkmal einzelner Praktiken ist oder ob er strukturell zu sozialen Praktiken überhaupt gehört. ${ }^{41}$

5. Bevor diese Frage geklärt werden kann, soll kurz skizziert werden, wie sich aus diesem Vorschlag ein umfassender Verdinglichungsbegriff ergibt, der alle vier zu Beginn dieses Aufsatzes genannten Aspekte umfasst.

Am einfachsten ist die vorgeschlagene Analyse auf den Fall der Verdinglichung sozialer Prozesse und Verhältnisse anzuwenden. Wenn soziale Praktiken, zu deren „Gelingensbedingungen“ eine bestimmte Form von Reflexivität gehört, diese durch ihre Normen zweiter Ordnung verhindern, dann werden der Ablauf dieser Praktiken, die in ihnen involvierten Beziehungen und die Praxis selbst für ihre Mitglieder unzugänglich und scheinbar unvermeidbar. Diese praktische Unzugänglichkeit rechtfertigt zudem scheinbar die Überzeugung, dass der Charakter dieser Beziehungen und Prozesse unvermeidbar, objektiv oder natürlich sei.

Da im Rahmen verdinglichter Praktiken auch die Bedeutung der Eigenschaften von Objekten unzugänglich wird, kann zudem erklärt werden, was es heißt, von der „Verdinglichung von Dingen“ zu sprechen. Wenn die Eigenschaften von Objekten scheinbar ohne Bezug auf die Einbettung unseres Handelns in Praktiken existieren, dann scheinen sie vollständig „objektiv“ zu sein. Wir sind ihnen gegenüber ohne Einfluss, und sie werden uns fremd. Damit verselbstständigt sich aber auch die Bedeutung, die wir Objekten in unseren Praktiken zuschreiben, und damit die Rolle dieser Objekte selbst. Die verdinglichte „Gegenständlichkeitsform“, von der Lukács spricht, ist also die Form, die Objekte annehmen, deren Eigenschaften zumindest teilweise sozialen oder institutionellen Charakter haben, wenn die übergreifenden Regeln der Praxis es nicht erlauben, die sozialen Praktiken und Institutionen als sozial konstituiert zu behandeln, in denen ihnen diese Eigenschaften zugeschrieben werden. Diese „Gegenständlichkeitsform“, die der Unzugänglichkeit und damit Unveränderbarkeit einer Praxis entspricht, steht in einem systematischen Zusammenhang mit der passiven, rein strategischen, „kontemplativen“ Haltung der Subjekte gegenüber ihren Praktiken.

Zum Abschluss kann zumindest angedeutet werden, wie vor diesem Hintergrund die Phänomene der Verdinglichung der eigenen Person und der Verdinglichung anderer Personen analysiert werden könnten: Es macht einen Unterschied für uns, ob wir unsere Identität, unsere Einstellungen, Gefühle und Entscheidungen als etwas begreifen, auf dessen soziale Bedeutung wir praktischen Einfluss haben. Die praktische Unzugänglichkeit einer Praxis, in der Aspekte unserer Identität Ausdruck finden, führt aus diesem Grund dazu, dass wir scheinbar passiv den Prozessen ausgeliefert sind, in denen unsere Einstellungen und Entscheidungen eine bestimmte soziale Bedeutung gewinnen. Das heißt, dass wir den sozial zugeschriebenen

41 Vgl. dazu Abschnitt IV. 
Qualitäten unserer eigenen Identität gegenüber einflusslos werden und sie uns fremd werden. Sobald sie für uns in dieser Weise zu „objektiven“ Gegebenheiten werden, werden wir außerdem dazu tendieren, ihnen gegenüber instrumentell-strategische Haltungen einzunehmen.

Auch Interaktionen mit anderen Personen verlieren ihre Bedeutung, wenn die Weise, in der uns diese anderen Personen als Anerkennungspartner in sozialen Praktiken begegnen, nicht mehr so erlebt wird, dass wir auf die Bedeutung dieser Interaktionen Einfluss nehmen können. Wenn diese Interaktionen von uns als ein objektives Geschehen behandelt werden müssen, das wir nur im Hinblick auf seine Folgen beurteilen können, kommen wir nicht umhin, eine distanzierte Haltung zu den Beziehungen einzunehmen, in denen andere Personen für uns Bedeutung gewinnen. Solch ein Prozess zwingt uns zwar nicht, andere Personen $\mathrm{zu}$ instrumentalisieren oder zu missachten, er hindert uns jedoch systematisch daran, die jeweils individuelle Persönlichkeit unserer Interaktionspartner voll wahrzunehmen, die den Anspruch dieser Personen auf Respekt begründet.

\section{IV.}

1. Es bleibt nun noch die Frage zu klären, woher die Normen, auf die sich die Verdinglichungskritik bezieht, eigentlich ihre Kraft gewinnen. Die bisher entwickelte Rekonstruktion weist das Defizit auf, dass sie anzunehmen scheint, dass schon die Natur sozialer Praktiken als solche einen Anspruch auf Reflexivität begründet. Eine solche Annahme würde aber nicht nur selbst eine verdinglichende Sichtweise darstellen, die eine sozialontologische Analysekategorie in den Rang einer zeitlosen Quelle menschlicher Verpflichtung erhöbe, sondern sie würde auch die Frage unbeantwortbar machen, wieso es sich bei der Verdinglichung um ein spezifisch modernes Phänomen handelt.

Dass es manche Praktiken zum Maßstab ihres Gelingens machen, dass sich die Teilnehmerinnen und Teilnehmer an der Praxis in der Praxis reflexiv auf die Praxis beziehen können müssen, ist jedoch kein Bestandteil einer universalen Form sozialer Praxis, sondern eben nur eine Norm bestimmter, historisch kontingenter Praktiken. Wie leicht zu sehen ist, gibt es zahlreiche Praktiken, die diese Norm nicht beinhalten. Dies gilt etwa für viele Spiele: Im Rahmen des Schachspiels selbst können wir die Schachregeln nicht verändern oder thematisieren. Die Schachregeln sehen solche Züge schlichtweg nicht vor, und dennoch wäre es offensichtlich müßig, das Schachspiel als ,verdinglicht“" zu bezeichnen.

Es besteht aber ein relevanter Unterschied zwischen Spielen und jenen Praktiken, bei denen ein reflexiver Bezug auf die Regeln ein Bestandteil ist, der nach ihren eigenen Normen zu ihrem Gelingen gehört: Wenn wir etwa an das Zusammenleben in einer Familie, an Erziehung, an ein Studium, an die Demokratie, an religiöse Traditionen, an den philosophischen Diskurs, an die Kunst oder an viele handwerkliche Praxisformen denken, gehört es faktisch konstitutiv zu diesen Praktiken, dass die prinzipielle Möglichkeit der Veränderung, Thematisierung, Kritik, Durchsetzung und auch Bewertung der Normen dieser Praktiken eine Bedingung des Gelingens dieser Praktiken darstellt.

Bei diesen Beispielen handelt es sich zudem nicht um randständige Praktiken, sondern um die zentralen Praktiken einer Lebensform, in der ganze Bündel von Praktiken systematisch miteinander verwoben sind. ${ }^{42}$ Die Regeln vieler alltäglicher Praktiken sind zwar nicht direkt praktisch zugänglich, wenn wir diese Praktiken isoliert voneinander betrachten. Sie sind aber

42 Diesen Begriff der „Lebensform“ entnehme ich der Studie von Jaeggi (2011); vgl. aber auch Lukács (1968), 362. 
so in ein Gewebe anderer Praktiken eingebunden, dass wir die Möglichkeit haben, uns auf diese Regeln im Rahmen anderer Praktiken legitim zu beziehen. Dies unterscheidet reflexive Praktiken von verdinglichten Praktiken, die sich mittels ihrer eigenen Regeln einer solchen Einbindung systematisch entziehen.

2. Verdinglichungskritik bezieht sich also auf eine bestimmte Form sozialer Praktiken, die zwar im engeren Sinne kontingent ist, die aber im historischen Kontext moderner Lebensformen keineswegs zufällig existiert. Es handelt sich dabei um Formen derjenigen reflexiven Praktiken, die das Prinzip in sich aufgenommen haben, dass wir nur an die Regeln gebunden sind, bei denen unsere praktische Akzeptanz die Grundlage ihrer Gültigkeit bildet.

Das heißt aber auch, dass Verdinglichungsphänomene ihre Ursache nicht nur in einem Verfall sozialer Praktiken, sondern auch in einer Ausweitung normativer Ansprüche haben können: Sobald eine soziale Praxis strukturell in die Lebensform der Moderne aufgesogen wird, werden vormals normativ nicht-widersprüchliche zu widersprüchlichen Erfahrungen. Eigenschaften von Handlungen, Beziehungen, Objekten oder Personen, die im Rahmen dieser Praktiken normative Bedeutung erlangen, können als unzugänglich erfahren werden, weil ihre „Form“ bestimmte Erwartungen frustriert. Diese Erwartungen existieren nicht zeitlos und kontextfrei. Sie werden vielmehr deshalb als legitim erfahren, weil sie von den impliziten Zielen und Standards dieser Praktiken gestützt werden. Das erklärt, wieso sich gerade in der Moderne, in der diese Ziele den Kern einer ganzen Lebensform bilden, Verdinglichungserfahrungen auf alle Bereiche ausdehnen.

3. Neben dieser Einordnung der normativen Basis der Verdinglichungskritik in eine historischsoziale Perspektive muss ihre Reichweite jedoch noch weiter begrenzt werden. Dies ist notwendig, um die oftmals Lukács zugeschriebene Position zu vermeiden, dass die Abwesenheit von Verdinglichung ein so transparentes Verhältnis zur gesellschaftlichen Realität erfordern würde, wie es nur ein kollektives Subjekt haben könnte, das dieser Realität mit der Freiheit eines Schöpfers gegenübertritt. Diese Vorstellung wäre nicht nur tendenziell totalitär, sie wäre auch so stark, dass die Verdinglichung als das unvermeidliche Schicksal jeder Gesellschaft zu gelten hätte und somit als Basis für die Gesellschaftskritik nutzlos wäre.

Dies ist jedoch keineswegs Lukács' Position. Vielmehr betont er, dass die Überwindung der Verdinglichung ,keine andere Form annehmen“ kann

„als die ununterbrochene [...] Tendenz, durch konkrete Beziehung auf die konkret zutage tretenden Widersprüche der Gesamtentwicklung, durch Bewußtwerden des immanenten Sinnes dieser Widersprüche [...] die verdinglichte Struktur des Daseins praktisch zu durchbrechen". ${ }^{43}$

Auch wenn uns seine Vorstellung dieser schrittweisen Aneignung der „Totalität“ durch eine revolutionäre Politik fremd geworden ist, so steht hinter diesen Bemerkungen doch die Einsicht, dass die durch die Verdinglichung verletzte Norm einer praktischen Zugänglichkeit der Objekte keineswegs dadurch erfüllt werden kann, dass ein kontingentes Hindernis ein für alle Mal aus dem Weg geräumt wird. Es muss sich dabei vielmehr immer um eine jeweils spezifische Reaktion auf Frustrationen legitimer Erwartungen handeln. Das Ziel kann nicht eine vollständige, transparente praktische Aneignung aller praktischen Relevanzen sein, sondern es muss um die jeweils kontextspezifische Aufhebung pathologischer Aneignungshindernisse gehen.

43 Ebd., 385. 
4. Wir können uns diesem Gedanken unabhängig von Lukács nähern, wenn wir die Frage stellen, was eine „,nicht-verdinglichte Praxis“ sein könnte. Es kann damit kaum die Forderung gemeint sein, dass jeder Teilnehmerin und jedem Teilnehmer einer Praxis in jeder Situation die Normen dieser Praxis vollständig im Modus der Veränderbarkeit, Thematisierbarkeit und Verfügbarkeit zugänglich sein müssen. Die Unmöglichkeit eines solchen Zugangs ist nämlich nur dann ein Problem, wenn in einer Situation legitimerweise erwartet werden kann, dass die entsprechenden Normen in der Praxis praktisch zugänglich werden können. Eine solche Situation ist aber nur dann gegeben, wenn ein erzwungenes Verbleiben der Normen im unhinterfragbaren Hintergrund ein praktisches Problem für die Akteure darstellt. Diese Bedingung ist aber normalerweise nur dann erfüllt, wenn die in Frage stehenden Normen entweder faktisch umstritten sind, wenn sie für die Betroffenen nicht tragbar sind oder wenn sie mit anderen Normen oder Zielen so in einem Konflikt stehen, dass die normative Integration der Praxis bedroht ist.

Das heißt umgekehrt, dass wir in denjenigen Fällen, in denen solche Konflikte nicht existieren, die Unzugänglichkeit von Normen keineswegs als Problem erleben müssen. Verdinglichung kann also nicht als die Verletzung einer Norm verstanden werden, die immer und überall die totale Transparenz von Praktiken einfordert. Verdinglichungskritik fordert nur die spezifische, an konkrete soziale Konflikte gebundene Zugänglichkeit der Normen derjenigen Praktiken ein, die durch ihre internen Ansprüche an diese Reflexivität als an ihre eigene Erfolgsbedingung gebunden sind.

\section{V.}

An der Verdinglichungskritik kann exemplarisch demonstriert werden, dass die Gesellschaftskritik nicht nur moralische Ansprüche, sondern auch strukturelle Standards des Gelingens von Praktiken berücksichtigen kann. Die Verdinglichungskritik verweigert sich aus diesem Grund der Unterscheidung zwischen Moral und Ethik. Sie betrifft das „gelingende Leben“, ohne - unter denjenigen Optionen, die mit einem modernen Selbstverständnis kompatibel sind - eine bestimmte Vorstellung des guten Lebens zu privilegieren.

Die Verdinglichungskritik nimmt nämlich nicht nur die moralische Frage in den Blick, welche Behandlung sich Personen wechselseitig schulden. Sie thematisiert auch die strukturellen Folgen von Veränderungen von Praxisformen. Dabei braucht sie keine universellen, unabhängig ableitbaren Normen ins Spiel zu bringen, sondern kann sich Praktiken in ihrer jeweils spezifischen Form nähern und ihre internen normativen Ansprüche in den Fokus rücken, deren Verletzung Verdinglichungserfahrungen anzeigen.

Dr. Titus Stahl, Johann Wolfgang Goethe-Universität, Institut für Philosophie, Grüneburgplatz 1, 60629 Frankfurt/M. 


\section{Literatur}

Anderson, E. S. (1990), Is Women's Labor A Commodity?, in: Philosophy \& Public Affairs, 19 (1), 71-92.

Arato, A. u. Breines, P. (1979), The Young Lukács and the Origins of Western Marxism, New York.

Berger, P. L. u. Luckmann, Th. (1977), Die gesellschaftliche Konstruktion der Wirklichkeit, Frankfurt/M.

Berger, P. L. u. Pullberg, S. (1965), Verdinglichung und die soziologische Kritik des Bewusstseins, in: Soziale Welt, 16 (1), 97-112.

Bloor, D. (1997), Wittgenstein, Rules and Institutions, New York.

Bourdieu, P. (1976), Entwurf einer Theorie der Praxis auf der ethnologischen Grundlage der kabylischen Gesellschaft, Frankfurt/M.

Brunkhorst, H. (1983), Paradigmakern und Theoriendynamik der Kritischen Theorie der Gesellschaft, in: Soziale Welt, 34 (1), 22-56.

Celikates, R. (2009), Kritik als soziale Praxis, Frankfurt/M.

Chari, A. (2010), Towards a Political Critique of Reification. Honneth, Lukács and the Aims of Critical Theory, in: Philosophy and Social Criticism, 36 (5), 587-606.

Dannemann, R. (1987), Das Prinzip Verdinglichung: Studie zur Philosophie Georg Lukács', Frankfurt/M.

Gould, S. (1994), The Mismeasure of Man, New York.

Habermas, J. (1995), Theorie des kommunikativen Handelns, Frankfurt/M.

Honneth, A. (2005), Verdinglichung, Frankfurt/M.

Jaeggi, R. (2003), Verdinglichung - ein aktueller Begriff?, in: Jahrbuch der Internationalen GeorgLukács-Gesellschaft, 3, 68-72.

Dies. (2011), Zur Kritik von Lebensformen, Berlin (im Erscheinen).

Jay, M. (1984), Lukács and the Origin of the Western Marxist Paradigm, in: ders., Marxism and Totality. The adventures of a concept from Lukács to Habermas, Berkeley.

Kant, I. (1977), Grundlegung zur Metaphysik der Sitten, Frankfurt/M.

Lukács, G. (1968), Die Verdinglichung und das Bewusstsein des Proletariats, in: ders., Geschichte und Klassenbewusstsein, in: ders., Werke, Bd. 2, Neuwied, 257-397.

Marx, K. (1962), Das Kapital, Bd. 1, Berlin.

Nussbaum, M. (2002), Konstruktionen der Liebe, des Begehrens und der Fürsorge, Stuttgart.

Pitkin, H. (1987), Rethinking reification, in: Theory and Society, 16 (2), 263-293.

Polanyi, K. (19789, The great transformation: politische und ökonomische Ursprünge von Gesellschaften und Wirtschaftssystemen, Frankfurt/M.

Reckwitz, A (2003), Grundelemente einer Theorie sozialer Praktiken, in: Zeitschrift für Soziologie, 32, 282-301.

Schatzki, T (1996), Social practices: A Wittgensteinian approach to human activity and the social, Cambridge.

Schatzki, T. u. a. (Hg.) (2001), The Practice Turn in Contemporary Theory, London.

Taylor, Ch. (1983), Hegel, Frankfurt/M.

Ders. (1975), Interpretation und die Wissenschaften vom Menschen, in: ders., Interpretation und Erklärung in den Wissenschaften vom Menschen, Frankfurt/M.

Ders. (1992), Was ist menschliches Handeln?, in: ders., Negative Freiheit? Zur Kritik des neuzeitlichen Individualismus, Frankfurt/M., 9-51.

Zurn, Ch. (2011), Social Pathologies as Second-Order Disorders, in: D. Petherbridge (Hg.), Axel Honneth. Critical Essays, 345-370, Leiden. 


\begin{abstract}
Although the critique of reification is a core commitment of critical theories, there is no widely accepted account of its normative foundation. In Lukács's original analysis, this foundation is provided by a strong concept of practice which is, however, not acceptable from a contemporary point of view. I argue that the systematic character of reification theory can only be upheld if this concept is replaced by a more intersubjective notion of normative practices. Reification can then be analysed as a secondorder pathology of social practices, as an inhibition of reflexivity by their normative shape. The normative justification of such critique, however, turns out to be more context-dependent than it is usually imagined.
\end{abstract}

